\title{
Forecasting future oil production in Norway and the UK: a general improved methodology
}

\author{
L. Fiévet ${ }^{\mathrm{a}, *}$, Z. Forró ${ }^{\mathrm{a}}$, P. Cauwels ${ }^{\mathrm{a}}$, D. Sornette ${ }^{\mathrm{a}}$ \\ ${ }^{a}$ Chair of Entrepreneurial Risks, ETH Zürich, Scheuchzerstrasse 7 (SEC F), CH-8092 Zürich, Switzerland
}

\begin{abstract}
We present a new Monte-Carlo methodology to forecast the crude oil production of Norway and the U.K. based on a two-step process, (i) the nonlinear extrapolation of the current/past performances of individual oil fields and (ii) a stochastic model of the frequency of future oil field discoveries. Compared with the standard methodology that tends to underestimate remaining oil reserves, our method gives a better description of future oil production, as validated by our back-tests starting in 2008. Specifically, we predict remaining reserves extractable until 2030 to be $188 \pm 10$ million barrels for Norway and $98 \pm 10$ million barrels for the UK, which are respectively $45 \%$ and $66 \%$ above the predictions using the standard methodology.
\end{abstract}

\section{INTRODUCTION}

$\mathrm{F}$ Orecasting future oil production has been a topic of active interest since the beginning of the past century because of oil central role in our economy. Its importance ranges from energy production, manufacturing to the pharmaceutical industry. As petroleum is a non-renewable and finite resource, it is primordial to be able to forecast future oil production. The fear of a global oil peak, beyond which production will inevitably decline, has been growing due to stagnating supplies and high oil prices since the crisis in 2008/2009 (Murray \& Hansen. 2013). As any industrialized country, Europe is strongly dependent on oil supply to maintain its economic power. In the nowadays difficult geopolitical environment, it is important to know how much of the oil needed in Europe will come from reliable sources. In the past, a big share has been coming from Norway and the U.K., two of Europe's biggest exporters. However, the U.K. already became a net importer in 2005 and Norway's production has been declining rapidly as well (Höök \& Aleklett, 2008).

The methodology behind forecasting future oil production has not evolved

\footnotetext{
${ }^{*}$ Corresponding author. Tel.: +41 446328379 .

Email addresses: lfievet@ethz.ch (L. Fiévet), zforro@ethz.ch (Z. Forró), pcauwels@ethz.ch (P. Cauwels), dsornette@ethz.ch (D. Sornette)
}

Preprint submitted to Elsevier 
much since M. King Hubbert, who in 1956 famously predicted that the U.S. oil production would peak around 1965-1970 (Hubbert, 1956). That prediction has proven itself to be correct. His main argument was based on the finiteness of oil reserves and to what amounts as the use of the logistic differential equation for the total quantity $P(t)$ of oil extracted up to time $t$

$$
\frac{d P}{d t}=r P\left(1-\frac{P}{K}\right)
$$

The logistic differential equation is characterized by an initial exponential growth, which then decreases to zero as the total oil extracted reaches saturation (no more oil is to be found). The parameter $r$ is commonly referred to as the growth rate, and $K$ as the carrying capacity (total quantity of oil that can be ultimately extracted). If $P(t)$ is the amount of oil extracted up to time $t$, then $f(t):=\frac{d P}{d t}$ is the oil production rate, the quantity that M. King Hubbert predicted with surprising accuracy to peak. From a methodological point of view, the Hubbert model has enjoyed a longstanding popularity in modeling future oil production given its simplicity. Various extensions have been studied by Brandt (2007) to account for multi-cycled or asymmetric production curves. The existing forecasts of future oil production use some form of the Hubbert model (Brecha, 2012; Laherrère, 2002; Lynch, 2002) or some economical model applied to aggregate production (Greiner et al., 2011), but none goes into the details of studying the underlying dynamics. The main reason for the lack of details is certainly the lack of available data.

In this article, a new methodology is introduced to forecast future oil production. Instead of taking the aggregate oil production profile and fitting it with the Hubbert curve or its variants (such as the multi-cyclic Hubbert curve), the production profile of each individual oil field is used. By extending their production into the future and extrapolating the future rate of discovery of new fields, the future oil production is forecasted by means of a Monte Carlo simulation. To demonstrate the generality of the methodology presented here, it is applied to two major oil producing countries with publicly available data: Norway (NPD) and the U.K. (GOV.UK, 2014).

\section{Methodology}

The idea behind the methodology is to model the future aggregate oil production of a country by studying the production dynamics of its individual constituents, the oil fields. The main benefit of this approach, compared to working directly with aggregate production data, is the possibility to forecast non-trivial oil production profiles arising from the combination of all the individual field dynamics. This ability reflects directly the fact that the total production is the sum of the contributions of each individual oil field in production. Thus modelling at the level of each field reflects more closely the reality and is likely 
Forecasting future oil production • July 2014

to be over-performing and more reliable, as we show below. In order to implement our approach, one must be able 1) to extend the oil production of each individual field into the future and 2) to extrapolate the rate of discoveries of new oil fields.

\section{II.1. Extending the oil production of individual fields}

The first step to predicting the future oil production of a country is to extrapolate the future production of existing fields and to estimate the error on this extrapolation. The data of the fields developed in the past shows a repeating asymmetric pattern. A good example is the Oseberg field shown in figure 1 . with a quick ramp up once the field is being developed, and then a peak or plateau before the oil field production starts decaying. The decay can take many different shapes and is governed by a variety of geological and economical factors. The goal of the fitting procedure is to capture as much the impact of these different factors as possible.

\section{II.1.1. Regular, irregular and new fields}

To be able to forecast the oil production of each individual field, regularity had to be found in the production's dynamics. Modeling the whole production profile from the beginning of extraction seems elusive due to the variety of the forms it can take. Fortunately, modeling the decay process is sufficient in order to extrapolate future oil production. A preliminary classification is necessary to achieve that goal. Figures 1,2 and 3 show that, independent of the country, oil fields can be classified into three main categories:

- Regular fields - Their decays show some regularity (see figure11);

- Irregular fields - The ones that do not decay in a regular fashion (see figure 2);

- New fields - The ones that do not decay yet. As such, there is no easy way to forecast their future oil production based on past data (see figure 3 ).

All the fields have been fitted using an automated algorithm, but the results have been subsequently checked visually to sort out the irregular fields which could not be fitted. As of January 2014, regular fields make up $85 \%$ and $87 \%$ of the number of fields and $94 \%$ and $71 \%$ of the total produced oil volume in Norway and the U.K. respectively. As such, being able to model them is crucial. To capture as many different decay dynamics as possible, the decay part of the oil production rate $f(t):=\frac{d P}{d t}$ has been fitted by the stretched exponential

$$
f(t)=f_{0} e^{-\left(\frac{t}{\tau}\right)^{\beta}} .
$$

The stretched exponential function has many advantages as it generalises the power law and can therefore capture a broad variety of distributions as shown 
by Laherrère \& Sornette (1998). Moreover, Malevergne et al. (2005) showed that the power law function can be obtained as an asymptotic case of the stretched exponential family, allowing for asymptotically nested statistical tests. As can be seen in figure 1. the stretched exponential (equation 2) is a good functional form to fit the decay process of regular fields.

For the minority of irregular fields, we assume no difference in the decay of production between giants and dwarfs, which has been modelled as follows: the decay time scale $\tau$ has been picked to be the average $\tau$ over the regular fields. Then $\beta$ has been fixed so that the sum of the field production over its lifetime be equal to the official ultimate recovery estimates, when such an estimate is available.

The minority of new fields, which did not yet enter their decay phase, cannot be extrapolated and will therefore be treated as new discoveries. The technical details of how to treat them as new discoveries are discussed in section II.2.3.

\section{II.1.2. Back-testing $\mathcal{E}$ Error}

To determine how well the extrapolation based on the stretched exponential predicts the future production, a complete back-testing has been performed on each field. A single back-test is made as follows:

- The production data $\left\{p_{0}, \ldots, p_{N}\right\}$ of the field is truncated at a certain date in the past $T \in\{0, \ldots, N\}$, where $T$ is the time counted in months since the production start of the field.

- The extrapolation of the oil production rate $f(t):=\frac{d P}{d t}$ is made based on the truncated data $\left\{p_{0}, \ldots, p_{T}\right\}$.

- The future production predicted by the extrapolation function $f(t)$ can be compared to the actual production from the date $T$ in the past up to the present $T_{f}=N$. The extrapolated total production can be computed as

$$
P_{e}(T)=\int_{T}^{T_{f}} f(t) d t
$$

and the relative error is given by

$$
e(T)=\frac{P_{e}(T)-\sum_{i=T}^{T_{f}} p_{i}}{P_{e}(T)},
$$

where both $P_{e}(T)$ and $e(T)$ are functions of the truncation time $T$.

Computing this back-test, for every month in the past since the field production started decaying, yields a plot showing the evolution of the relative error over time defined by expression (4). By construction, the relative error will tend to zero as the truncation time $T$ approaches the present. Nonetheless, it is a useful indicator for the stability of the extrapolation. As can been seen for the Oseberg 
field in figure 4 the relative error on future production remained fairly stable during the past decade.

From the complete back-test, we compute the average relative error

$$
\bar{e}=\frac{1}{N} \sum_{i=0}^{N} e(i)
$$

of the extrapolation made on the future production. Assuming that the relative errors are normally distributed around the average relative error, the standard deviation on the average relative error is given by

$$
\sigma_{e}=\sqrt{\frac{1}{N} \sum_{i=0}^{N}(e(i)-\bar{e})^{2}} .
$$

As the average relative error is often fairly constant, the extrapolation was corrected by the average relative error, that is, if the extrapolation consistently over-estimated the production by $10 \%$ during the back-test, the extrapolation was reduced by $10 \%$. This results in an extrapolated production $p(t)$, including a $1 \sigma$ confidence interval, given by

$$
p(t)=(1-\bar{e}) f(t) \pm \sigma_{e} .
$$

An example of such an extrapolation including a one standard deviation range is shown in figure 1 for the Oseberg field.

\section{II.1.3. Aggregate error}

Once the individual fields have been extrapolated using formula 7 , we compute the extrapolation of the oil production for the whole country. While it is straightforward to sum the extrapolations of the individual fields to obtain the expected production, some care has to be taken with respect to the confidence interval of the production at the country level.

As shown later in section III. the same extrapolation including a complete monthly back-test of total future production has been performed at the country level and the resulting relative error is much smaller than the average error observed on the individual fields. To account for this observation, the assumption made is that the relative error between individual fields is uncorrelated. While one could imagine that some inter-dependence could result from a coordinated response of supply to a sharp increase/decrease of demand, we have not observed this to be the case at a significant level. Therefore, the fields can be considered as a portfolio of assets with a return given by their extrapolation $p(t)$ (eq. 7) and a risk given by $\sigma_{\text {field }}$ (eq. 6). This means that the average standard deviation per field at the country level from the extrapolated production can be computed as

$$
\sigma_{\text {country }}^{2}=\frac{1}{\# \text { fields }} \sum_{\text {field } \in \text { fields }} \sigma_{\text {field }}^{2}
$$


Intuitively, this models well the fact that the uncorrelated errors among fields will mostly cancel out.

\section{II.2. Discovery rate of new fields}

Knowing the future production rate of existing fields is not enough as new fields will be discovered in the future. The model describing the discovery rate of new fields should satisfy two fundamental observations.

1. The rate of new discoveries should tend to zero as time goes to infinity. This is a consequence of the finiteness of the number of oil fields.

2. The rate of new discoveries should depend on the size of the oil fields. As of today, giant oil fields are discovered much less frequently than dwarf oil fields.

\section{II.2.1. Discoveries modeled as logistic growth}

A natural choice for such a model is a non-homogenous Poisson process. The Poisson process is a process that generates independent events at a rate $\lambda$. It is nonhomogeneous if the rate is time-dependent, $\lambda \rightarrow \lambda(t)$. The standard way to measure $\lambda(t)$ is to find a functional form for $N(t)$, the statistical average of the cumulative number of events (discoveries) up to time $t$. Then, $\lambda(t)$ is simply a smoothed estimation of the observed rate $\frac{d N(t)}{d t}$. Figure 5 shows $N(t)$ for Norwegian fields classified according to their size in two classes, dwarfs and giant fields. The logistic curve is a good fit to the data (integral form of equation (1). This implies that after an initial increase, the rate of new discoveries reaches a peak followed by a decrease until no more oil fields are to be found, consistent with our fundamental observations. This same approach has already been successfully applied by Forró et al. (2012) to estimate the number of daily active users on Zynga.

As the discovery and production dynamics are not independent of the field size, the fields have been split into two groups: dwarfs and giants. Unfortunately, the two logistic curves thus obtained are highly sensitive to the splitting size. This results from the major issue, when fitting a logistic curve to data, that the carrying capacity can not be determined if the data does not already exhibit the slowdown in growth towards the carrying capacity. However, it is mentioned in the literature that often dwarf fields have already been discovered a long time ago, but their production has been postponed for economical reasons (Lynch. 2002, p. 378). Therefore, it is expected that the large oil fields have mostly been found and produced, and that future discoveries will mostly be made up of dwarf fields. Consequently, the splitting size has been picked as small as possible in order to maximize the number of giant fields but large enough to avoid recent discoveries. Our definition is thus:

- Dwarfs: Fields which produced less than $50 \cdot 10^{6}$ barrels. 
- Giants: Fields which produced more than $50 \cdot 10^{6}$ barrels.

We note that this definition differs by a factor 10 from the more standard one, for which oil fields with an ultimate recoverable resource of 0.5 billion barrels $(\mathrm{Gb})$ or higher are classified as giants, while oil fields with smaller URR are considered to be dwarfs (Höök \& Aleklett, 2008).

The resulting plot shown in figure 5 pictures the dynamics: giant oil fields have mostly been found while the discovery process for dwarf fields is still ongoing. The logistic growth curve fit to the giant discoveries is well constrained, however the fit for the dwarfs is poorly constrained. As can be seen in figure 5 . the carrying capacity $K$ of the logistic growth model is not well constrained by the available data. A large spectrum of values for $K$ can lead to an equally good fit of the data. We have not taken into account the possible effect that newly discovered dwarf fields could become smaller and smaller until the new fields have a size that is too small to be economical to drill.

There are in fact two competing effects that are likely to compensate each other. On the one hand, figure 6 shows the complementary cumulative distribution function (CCDF) of known oil field sizes $S$ from Norway and the UK. Two salient properties can be observed. First, the tails of the distributions are well described by power laws

$$
\operatorname{CCDF}(S) \sim \frac{1}{S^{1+\alpha}}
$$

with exponent $\alpha=1.2 \pm 0.1$ for Norway and $\alpha=1.4 \pm 0.1$ for the UK. The fact that the estimations of the exponents $\alpha$ are larger than 1 implies that the cumulative oil reserves are asymptotically controlled by the largest fields, and not the small ones (Sornette, 2004). However, the fact that the exponents $\alpha$ are rather close to 1 (which is called "Zipf law") would make the many small oil field contributing significantly in total. This brings us to the second important feature exhibited by figure 6, namely the roll-overs of the CCDFs for small oil fields, likely due to an under-sampling of the data. Indeed, as for most data sets involving broad distributions of sizes such as oil fields, the distributions are in general incomplete for the small events due to the non exhaustive sampling. This incompleteness raises the spectre that our extrapolations might be grossly underestimating the large potential contributions to the total reserves of the many small yet undiscovered small oil fields. Assuming that the power law (9) would hold for smaller fields down to size of 1 Million barrels leads to a number of such fields 10 to 100 times larger than presently known.

But there is another key factor that needs to be considered, namely the fact that small oil fields are not economically viable for exploitation, which leads to an effective truncation in the distribution (9) relevant for the estimation of recoverable oil. Taking the data from the NPD providing the yearly investments broken by fields in Norway, let us consider the illustrative case of the field GAUPE. The investment spent to develop its exploitation was $\$ 380 \mathrm{M}$, while its 
estimated size is $\simeq 1.2 \mathrm{Mb}$, which corresponds approximately to a total market value of $\$ 120 M$ at $\$ 100$ per barrel. It is thus not a surprise that investment to exploit this field was interrupted in 2013. Figure 7 shows the total estimated revenue divided by investment as a function of the estimated ultimate recovery for a number of small oil fields. It can be inferred that oil fields of sizes smaller than about $10 \mathrm{Mb}$ are not economically viable as long as the market oil price does not grow much higher than $\$ 100$ per barrel. This implies that, notwithstanding the large number of unknown small oil fields, economic considerations oblige us to neglect the small oil fields, therefore providing a justification of our procedure. In fact, economic constraints may lead to cap the carrying capacity at a value smaller than the one shown in figure 5 .

To address these issues from a more solid angle, the method described in the next section II.2.2 has been used to compute the probability of different carrying capacities.

\section{II.2.2. Likelihood function for the number of discoveries}

To overcome the poor constraint on the carrying capacity $K$ obtained from the fitting procedure for dwarf fields, a method already used by Smith (1980) has been implemented. This method makes the following two postulates:

1. "The discovery of reservoirs in a petroleum play can be modeled statistically as sampling without replacement from the underlying population of reservoirs."

2. "The discovery of a particular reservoir from among the existing population is random, with a probability of discovery being dependent on (proportional to) reservoir size."

The fields are split into $J$ size bins denoted $S_{1}, \ldots, S_{J}$ occurring with frequency $n_{1}, \ldots, n_{J}$. Each discovery is considered as a step $i$ at which a field of size $I(i) \in$ $\left\{S_{1}, \ldots, S_{J}\right\}$ is found and $m_{i j}$ denotes the number of fields of size $j \in\{1, \ldots, J\}$ discovered before the $i^{t h}$ step. Then, the probability that the discovery at step $i$ is of size $j$ can be expressed as

$$
P\left(I(i)=S_{j}\right)=\frac{\left(n_{j}-m_{i j}\right) \cdot S_{j}}{\sum_{k=1}^{J}\left(n_{k}-m_{i k}\right) \cdot S_{k}} .
$$

The likelihood $L$ for a complete sequence of $N$ discoveries $\{I(1), \ldots, I(N)\}$ can then be expressed as

$$
L=\prod_{i=1}^{N} \frac{\left(n_{I(i)}-m_{i I(i)}\right) \cdot S_{I(i)}}{\sum_{j=1}^{J}\left(n_{j}-m_{i j}\right) \cdot S_{j}} .
$$

The unknown parameters are the number of fields $n_{1}, \ldots, n_{J}$, whose likelihood can now be estimated based on the existing discoveries. Using a brute force 
approach, the entire space of plausible values for the variables $n_{1}, \ldots, n_{J}$ has been sampled. The values $n_{j}$ have been sampled between the number of existing fields $m_{N j}$ in the bin $j$ and up to a value $n_{j}^{\text {upper }}$, such that the scenario with the largest likelihood according to equation 11 has $n_{j}^{\text {max }}=n_{j}^{\text {upper }} / 2$ fields in the bin $j$. Subsequently, the likelihood of each scenario (value of the tuple $n_{1}, \ldots, n_{J}$ ) has been normalized such that the total likelihood of all generated scenarios equals one.

For the analysis of the discoveries of the North Sea oil fields, the number of size bins has been fixed to $J=2$ splitting between dwarfs (1) and giants (2) as described in section II.2.1.

The results shown in table 1 are coherent with the intuitive expectation that discovering a new giant field is unlikely and that future discoveries will mostly be made up of dwarf fields. The likelihoods obtained for the carrying capacities of dwarfs and giants have been used to constrain the logistic curve fitted to the discoveries. Figure 5 shows a sample of fitted logistic curves, each curve being weighted by the likelihood of its carrying capacity given by equation (11).

\section{II.2.3. Future production from discoveries}

We now compute an expected oil production coming from future discoveries, which requires to combine the steps described in sections II.2.1 and II.2.2.

The method described in section II.2.2 yields probabilities for the total number of fields (including the not yet discovered fields) in each size bins (called a scenario). However, this likelihood method does not give the time distribution of future discoveries. We propose to use the likelihood function to generate scenarios with their respective occurrence probability.

For a given scenario, the carrying capacity $K$ (= total number of fields) is given for each size class. This is useful to resolve the instability in fitting the logistic curve to the number of discovered fields. The time distribution of the discoveries is then given for each size class by the fitted logistic curve.

The actual size of a newly discovered field is generated according to the size distribution of the existing fields in its size class. The probability distribution function of field sizes in a given size bin has been fitted by a stretched exponential function.

The production curve is computed based on the average production curve of all existing fields in the same size category. The production curves of the existing fields have all been normalized to a total production of one and then have been averaged. This yields the typical production profile including a one sigma confidence interval. For a new field, this typical production curve is than multiplied by the size of the field.

Superposing the production curves results in the expected production curve from future oil fields for a given scenario.

As the total parameter space is too large to be sampled entirely, a Monte Carlo technique is applied to compute the expected production with confidence 
Forecasting future oil production • July 2014

interval from future discoveries. In a nutshell, the algorithm works as follows:

1. Draw a scenario (total number of fields in each size bin) based on its probability according to the likelihood function (11). This is done by generating a random number $r$ between 0 and 1, and computing the scenario that is mapped to $r$ by the cumulative distribution function of all scenarios.

2. Compute the time distribution of new discoveries by fitting a logistic curve for each size class.

3. For each discovery, generate a size and the resulting production curve based on the size distribution and production curves of existing fields.

4. Superpose all the production curves.

5. Repeat and average over all drawn scenarios.

The result is the expected production curve of future oil field discoveries. The distribution of generated scenarios yields the confidence interval.

Last but not least, it has to be defined how the expected production from these future oil field discoveries is added to the extrapolated production from existing fields. The start of the simulation of new discoveries does not match up with the date of the latest production data, the reason being that the new fields (defined in section II.1.1), which are already discovered but did not yet enter the decay phase, are not taken into account in the simulation as their final size is not known. The only meaningful way of treating the new fields is to consider them as a discovery. Therefore, the starting point in time of the simulated production resulting from new discoveries has been choosen as the date in the past where it matches the current production from new fields. In order words, the extrapolated production from regular and irregular fields added to the production from simulated future discoveries (which is shifted into the past as to match the production from new fields) must be equal to the current (latest available data) total production from all fields.

\section{RESULTS}

Based on the methodology described in section II.2, simulating future oil production was straightforward. For each country, the existing oil field productions were extrapolated and the future discoveries were simulated. Figure 8 shows the average of 1000 simulations. For each country, the non-symmetric shape of the production dynamics, which contradicts the prediction based on Hubbert's standard approach, is immediately noticeable.

The results in table 2 show a striking difference between the extrapolation of the fit and the Monte-Carlo model forecast. According to the fit (extrapolation of aggregate production), Norway's future oil production would decay much 
faster than in the Monte-Carlo case. The remaining reserves estimated with the Monte-Carlo methodology are $45 \%$ larger then the estimate from the fit. This difference originates from two different effects:

- The sum of the forecast of the individual existing fields is larger than the extrapolation of the aggregate production.

- The extrapolation of the aggregate production does not capture well the discovery process of dwarf fields.

In the U.K., oil production faced a change of regime during the early nineties due to technological innovation, giving rise to the inverted "w shape" of the oil production profile. However, this has not been an issue for us to extrapolate the decay of production starting at the second peak. The difference between the Hubbert-based methodology and the Monte-Carlo one is very similar to the Norwegian case. The former underestimates the remaining oil reserves by about $66 \%$ compared to the latter.

Which of the two models is more trustworthy? Clearly, the implications in adopting one methodology over the other are significant. The only way to answer this question is to back-test them. In other words: "What would each of the models have predicted, had they been used in the past?" The next section addresses this question and presents the validation step of our approach.

\section{VALIDATION}

For both countries, namely Norway and the U.K., a back-test using the data truncated in 2008 has been made. Before that date, too many of the giant fields have not entered their decay phase for a sufficiently long time to apply the extrapolation algorithm. Figure 9 shows the results of these tests. Comparing the forecast of both models, with the oil production of the subsequent 6 years, shows that the predictive power of the Monte-Carlo model is significantly better than a simple extrapolation of the aggregate past production. The MonteCarlo model is found essentially right on target, while the extrapolation of past production ("fit") is under-estimating the realised production by $19 \%$ and $16 \%$ respectively for Norway and the U.K. The following table 3 summarizes the difference between the two approaches for the back-testing period.

While the error of the extrapolation of the past oil production method ("fit") is not dramatic over these six years from 2008 to 2014, the difference between it and the Monte-Carlo approach becomes huge from 2014 to 2030, as shown in table 4

The simple extrapolation decays too fast and entirely misses the fat tails in the decay process of individual fields and the new discoveries. Moreover, it must be noted that the simple extrapolation changed massively between the back-test in table 4 and the current fit in table 2 (520\% for Norway and 236\% for the U.K.). In other words, the simple extrapolation is very unstable in its 
Forecasting future oil production • July 2014

forecast, while the Monte-Carlo forecasts remains very consistent (less than $10 \%$ change).

As can be seen in figure 9. the actual production of Norway during the back-testing period remained entirely within the quite narrow $1 \sigma$ interval of the Monte-Carlo methodology, while totally breaking out of the $1 \sigma$ interval of the simple extrapolation. For the U.K. the Monte-Carlo methodology only performs slightly better when considering the confidence interval, and the confidence interval is much larger due to the uncertainty on future discoveries and their production profile.

\section{CONCLUSION}

We have presented a Monte-Carlo based methodology to forecast future oil production. By extending the oil production of current fields into the future and modeling the discovery rate of new fields, the future oil production of Norway and the U.K. could be forecasted. These forecasts are significantly different from the ones obtained with a standard extrapolation. Indeed, our model forecasts $45 \%$ to $66 \%$ more remaining oil reserves than the standard extrapolation. The back-test performed on the time period between 2008 and 2014 confirmed that the Monte-Carlo based model better captured the production dynamics.

The results suggest that it is highly likely that the decay of Norwegian and U.K. oil production will be much slower then one would expect from a standard extrapolation. Nonetheless, to maintain current levels of oil consumption in the European Union, more of it will have to be imported from outside Europe, as the imports from Norway will vanish (currently accounting for $11 \%$ of E.U. oil imports (European Commission)) and the U.K. will need to import more oil.

As shown in table 5, at constant consumption, the Monte-Carlo model predicts that in 2030 the E.U. with Norway will need to increase its oil imports by 1.3 million barrels. These imports will most likely have to come from outside Europe, except for non-standard oil sources yet to be developed.

The present methodology can be applied to many other countries and geological areas, as well as updated at the level of the global oil production. Extensions to include the new wave of shale oil and non-standard oil can in principle be considered and constitute an interesting domain of application of our methodology for the future.

Acknowledgements: This work derives from a Master thesis at ETH Zurich authored by Marc A. Del Degan (July 2012) under the supervision of the authors, entitled "Analysis of peak oil with focus on Norwegian oil production" (http:// www.er.ethz.ch/publications/MAS_Thesis_DelDegan_final_July12.pdf), which itself derived from the methodology of pricing social network companies such as Zynga developed by three of the authors Forró et al. (2012). 
Forecasting future oil production • July 2014

\section{REFERENCES}

Brandt, A. R. (2007). Testing Hubbert. Energy Policy, 35, 3074-3088. doi 10.1016/ j.enpol.2006.11.004.

Brecha, R. J. (2012). Logistic curves, extraction costs and effective peak oil. Energy Policy, 51, 586-597. doi $10.1016 /$ j.enpol.2012.09.016

European Commission (2014). EU Crude Oil Imports. URL: http://ec.europa. eu/energy/observatory/oil/import_export_en.htm.

Forró, Z., Cauwels, P., \& Sornette, D. (2012). When games meet reality: is Zynga overvalued? The Journal of Investment Strategies, 1, 119-145. URL: http://ideas.repec.org/p/arx/papers/1204.0350.html

GOV.UK (2014). Oil and gas: field data. URL: https://www.og.decc.gov.uk/ pprs/full_production.htm.

Greiner, A., Semmler, W., \& Mette, T. (2011). An Economic Model of Oil Exploration and Extraction. Computational Economics, 40, 387-399. URL: http://link. springer.com/10.1007/s10614-011-9272-0 doi:10.1007/s10614-011-9272-0.

Höök, M., \& Aleklett, K. (2008). A decline rate study of Norwegian oil production. Energy Policy, 36, 4262-4271. doi $10.1016 /$ j.enpol.2008.07.039

Hubbert, M. K. (1956). Nuclear energy and the fossil fuels. Drilling and Production Practice, (p. 36). URL: http://www.hubbertpeak.com/hubbert/1956/1956.pdf.

Laherrère, J. (2002). Forecasting future production from past discovery. International Journal of Global Energy Issues, 18, 218-238.

Laherrère, J., \& Sornette, D. (1998). Stretched Exponential Distributions in Nature and Economy: "Fat Tails" with Characteristic Scales. Physical Journal B-Condensed Matter and Complexe Systems, 2, 525-539.

Lynch, M. C. (2002). Forecasting oil supply: theory and practice. Quarterly Review of Economics and Finance, 42, 373-389.

Malevergne, Y., Pisarenko, V. F., \& Sornette, D. (2005). Empirical Distributions of Log-Returns : between the Stretched Exponential and the Power Law? Quantitative Finance, 5, 379-401. arXiv:0305089v1.

Murray, J. W., \& Hansen, J. (2013). Peak Oil and Energy Independence : Myth and Reality. Eos, Transactions American Geophysical Union, 94, 245-246. URL: http://doi.wiley.com/10.1002/2013EO280001.

Norwegian Petroleum Directorate (2014). Factpages. URL: http://factpages. npd.no/factpages/ 
Smith, J. L. (1980). A Probabilistic Model of Oil Discovery. The Review of Economics and Statistics, 62, 587-594.

Sornette, D. (2004). Critical Phenomena in Natural Sciences, Chaos, Fractals, Selforganization and Disorder: Concepts and Tools. (2nd ed.). Heidelberg. 


\section{Production of the field OSEBERG}

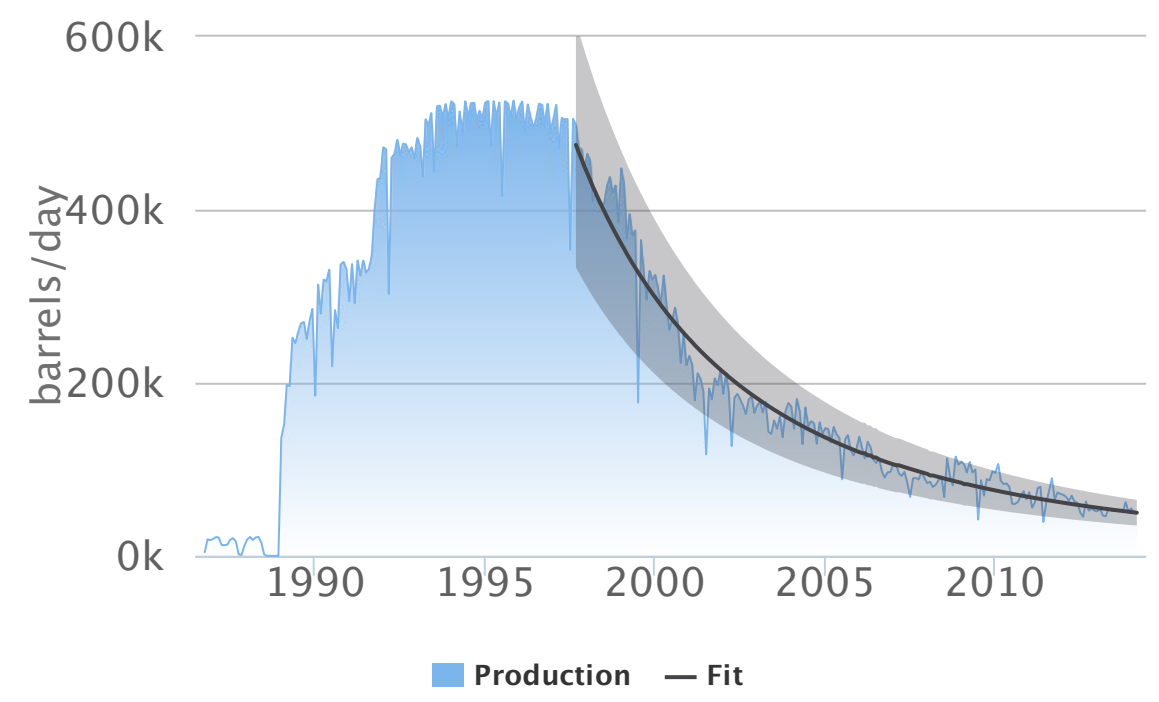

Figure 1: Example of the time evolution of the oil production per day of a regular field, parameterised beyond the peak in the decay regime by the stretched exponential function 2. with $\beta=0.66 \pm 0.01$ and $\tau=-55 \pm 1$ months, shown with the black line. The one standard deviation given by expression (7) is represented by the grey band. 
Forecasting future oil production • July 2014



Figure 2: Example of a irregular field. 


\section{Production of the field SKARV}

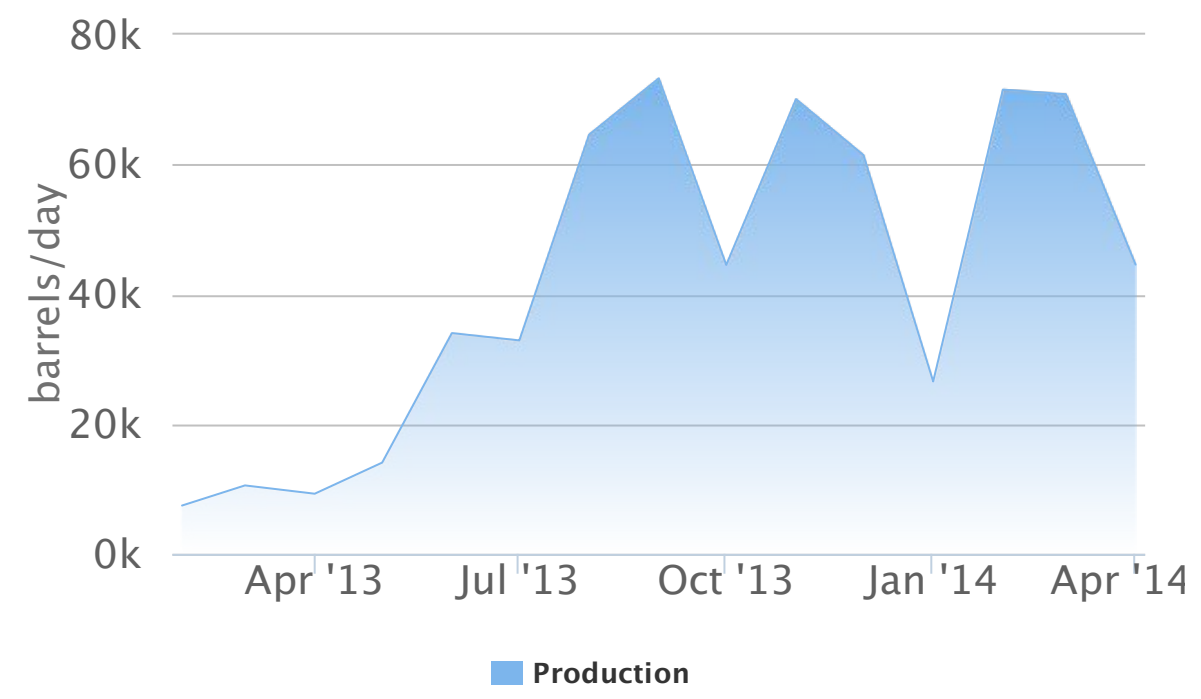

Figure 3: Example of a new field. 


\section{OSEBERG - Error on future total production}

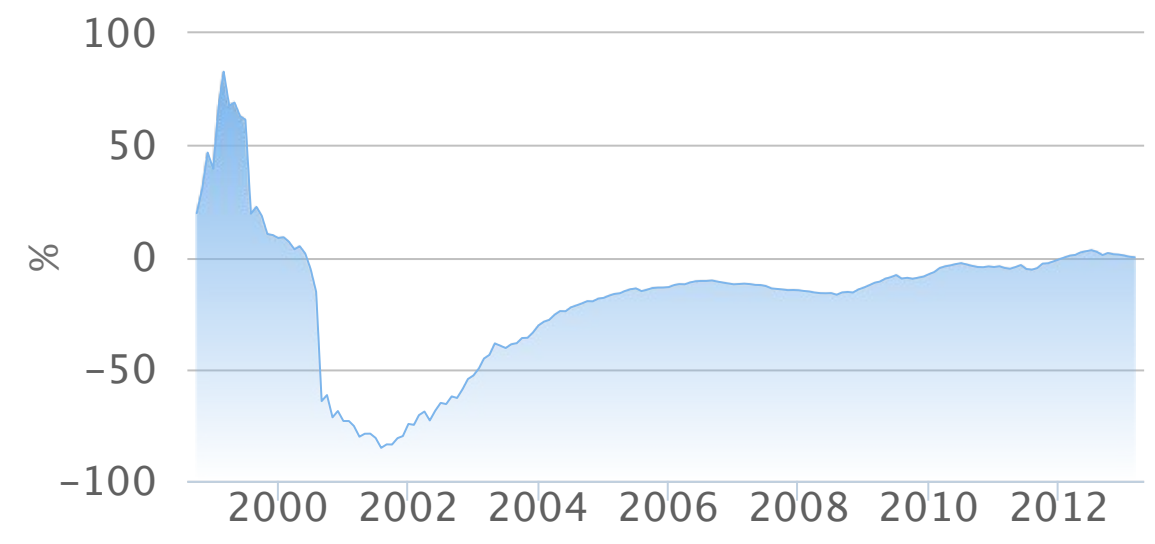

Fit error

Figure 4: Oesberg field - Relative error defined by expression (4) of the predicted total production from time $t$ indicated in the abscissa until 2014. One can observe that the predicted future total production is over-estimated by as much as 70\% in 1999, then underestimated by the same amount in 2002, while the forecast errors remain smaller than $20 \%$ since 2004 . 


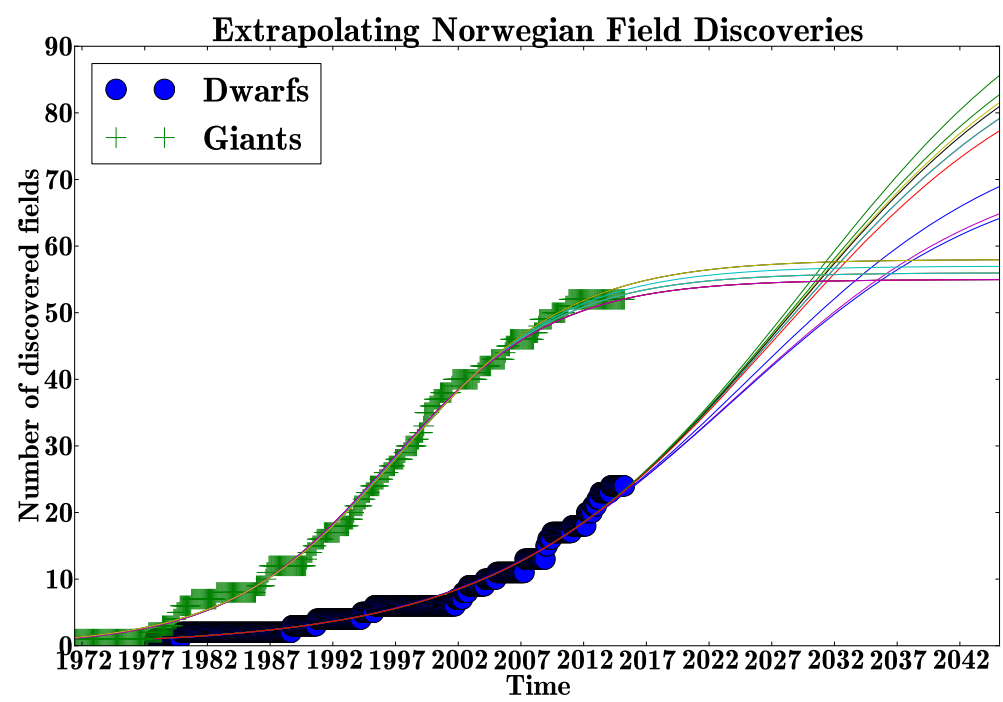

Figure 5: Logistic fit of the function solution of expression (17) to the number of discoveries for Norway. The discovery rate of new oil fields is dependent on their size as explained in the main text. 


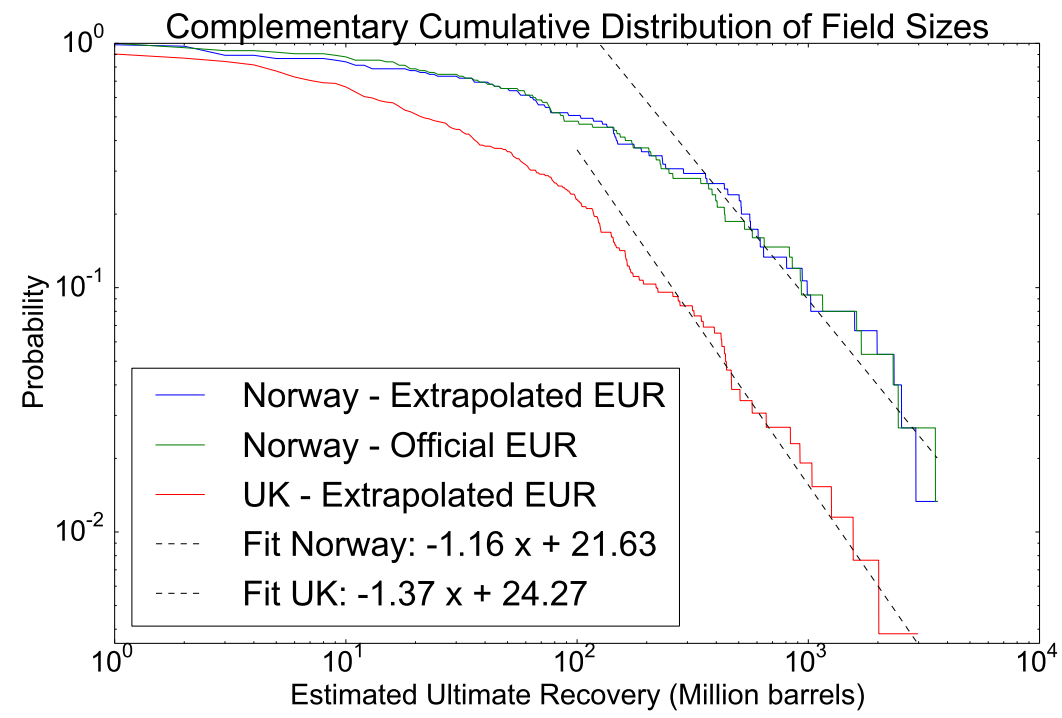

Figure 6: Complementary cumulative distribution function (CCDF) of known oil field sizes $S$ from Norway and the UK. The two dashes lines visualise the power law behaviour (9) of the tail of the distributions. 
Forecasting future oil production • July 2014

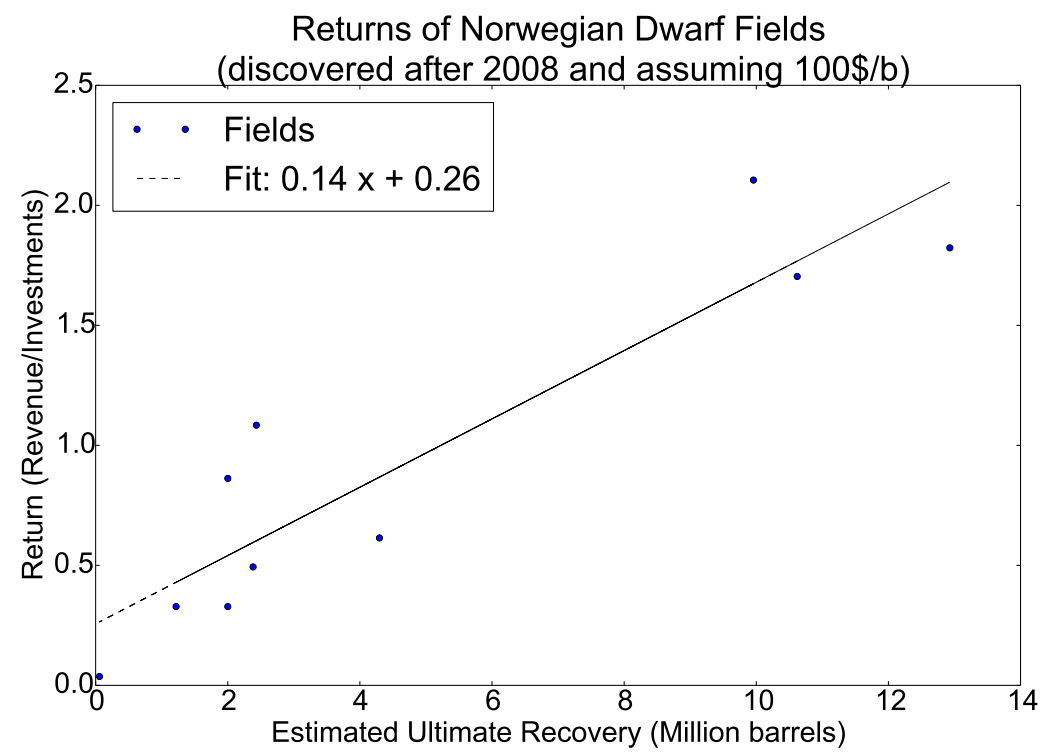

Figure 7: Total estimated revenue divided by investment as a function of the estimated ultimate recovery for a number of small oil fields. 


\section{Norwegian oil production and forecast}

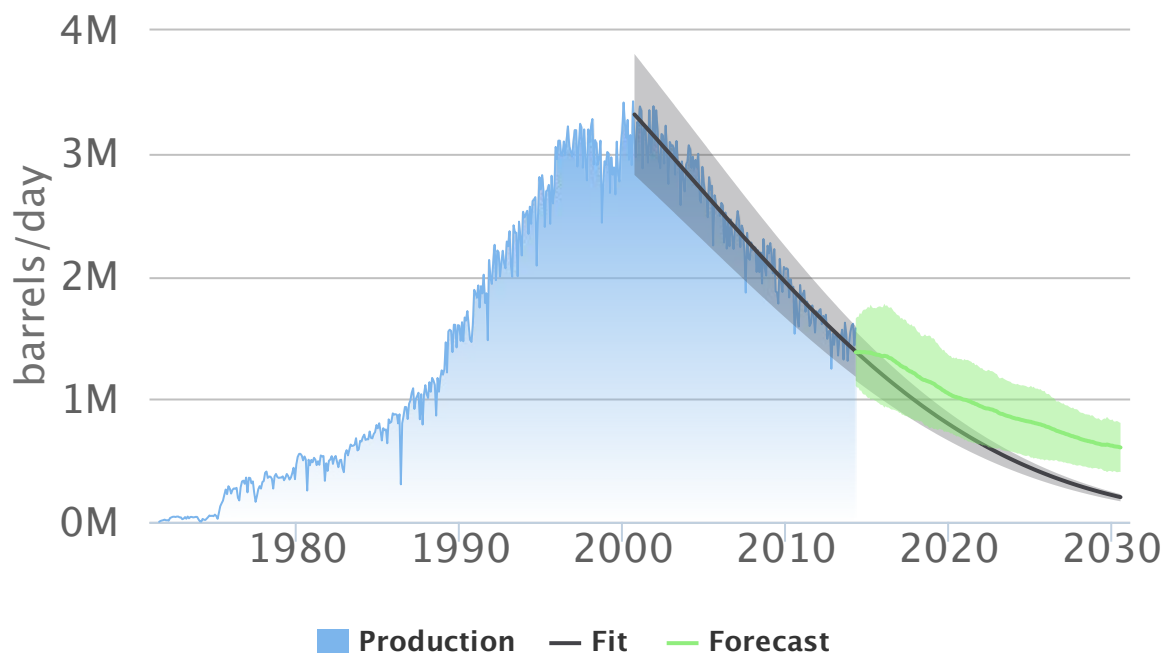

\section{U.K. oil production and forecast}

$$
\text { 4M }
$$

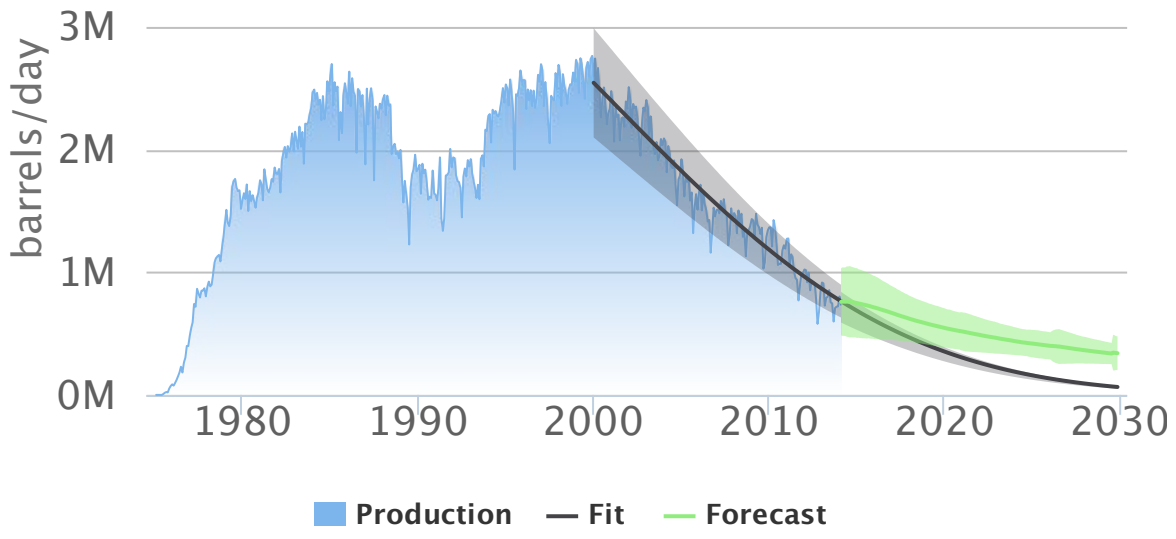

Figure 8: Monte-Carlo (green upper continuous line with standard deviation bank starting in 2014 onward) and fit forecast based on past production data (lower line and grey one standard deviation band) for Norway (top) and the U.K. (bottom). In both cases, the Monte-Carlo model forecasts a significantly slower decay than the fit by taking into account that new fields will come in production. 


\section{Norwegian oil production and forecast}

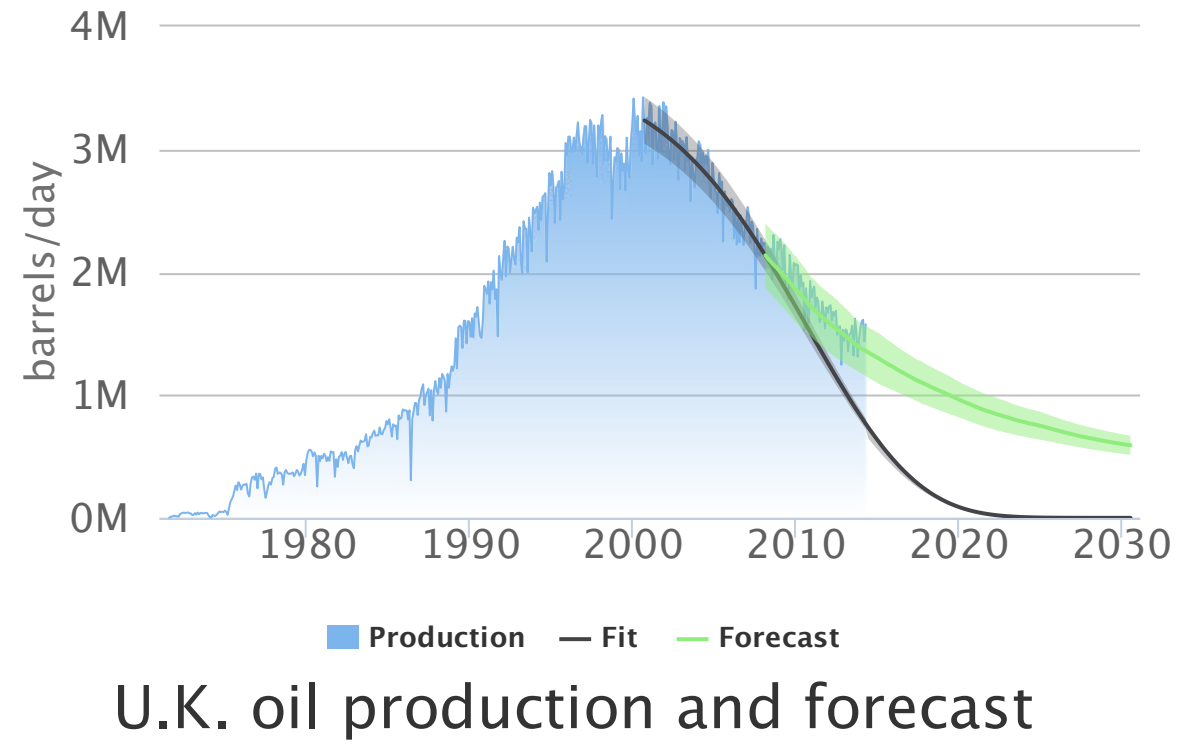

$$
\text { 4M }
$$

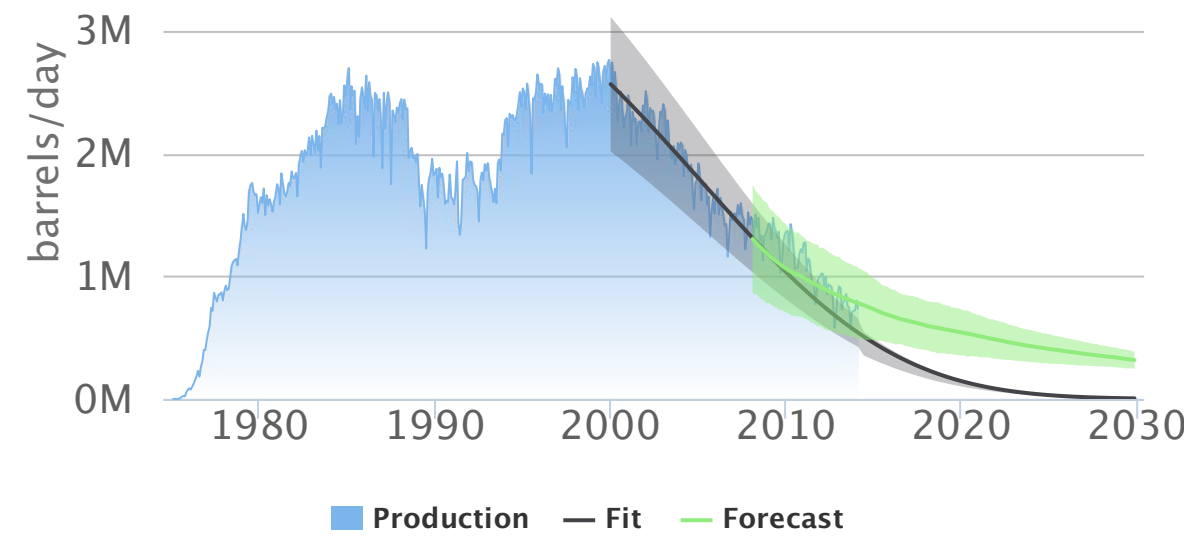

Figure 9: Monte-Carlo (green upper continuous line with standard deviation bank starting in 2014 onward) and Hubbert forecast based on past production data up to 2008 (lower line and grey one standard deviation band) for Norway (top) and the U.K. (bottom). The results can be compared with the subsequent oil production (blue area). In both cases, the Monte-Carlo methodology is more precise. 
Forecasting future oil production • July 2014

Table 1: Likelihood estimation for the number of dwarf (1) and giant (2) fields. For Norway, our logistic fit suggests that up to two new giant fields could be ultimately discovered. For the U.K., the prediction is more bleak, suggesting that the most probable scenario is that one giant field will be found (which is most likely already discovered but classified as a new field).

\begin{tabular}{lllll} 
& $m_{N 1}$ & $n_{1} \pm \sigma_{1}$ & $m_{N 2}$ & $n_{2} \pm \sigma_{2}$ \\
\hline Norway & 24 & $88.4 \pm 10.0$ & 52 & $56.4 \pm 1.6$ \\
U.K. & 162 & $208 \pm 11$ & 99 & $100 \pm 0.4$ \\
\hline
\end{tabular}


Forecasting future oil production • July 2014

Table 2: Remaining oil reserves until 2030 in barrels predicted by the extrapolation of the Fit of the past country production, and predicted by the Monte-Carlo Model. The relative difference between these two predictions is defined by $\Delta=\frac{\text { Model-Fit }}{\text { Fit }}$.

\begin{tabular}{lllr} 
& Fit (barrels) & Model (barrels) & $\Delta$ \\
\hline Norway & $130 \cdot 10^{6}$ & $188 \cdot 10^{6}$ & $45 \%$ \\
U.K. & $59 \cdot 10^{6}$ & $98 \cdot 10^{6}$ & $66 \%$ \\
\hline
\end{tabular}


Forecasting future oil production • July 2014

Table 3: Extrapolation of past oil production ("fit") and prediction using the Monte-Carlo model are used on the data set truncated in 2008. Their forecast for the period 2008-2014 is compared to the actual realised production.

\section{Actual (barrels) Fit (barrels) Model (barrels)}

\begin{tabular}{llll}
\hline Norway & $133 \cdot 10^{6}$ & $108 \cdot 10^{6}$ & $130 \cdot 10^{6}$ \\
U.K. & $79 \cdot 10^{6}$ & $66 \cdot 10^{6}$ & $75 \cdot 10^{9}$ \\
\hline
\end{tabular}


Forecasting future oil production • July 2014

Table 4: Remaining oil reserves forecasted for the period 2014-2030 when using the data truncated in 2008, according to the extrapolation of past oil production ("fit") and the Monte-Carlo model. The relative difference between these two predictions is defined by $\Delta=\frac{\text { Model-Fit }}{\text { Fit }}$.

\begin{tabular}{lllc} 
& Fit (barrels) & Model (barrels) & $\Delta$ \\
\hline Norway 08 & $25 \cdot 10^{6}$ & $171 \cdot 10^{6}$ & $-584 \%$ \\
U.K. 08 & $25 \cdot 10^{6}$ & $91 \cdot 10^{9}$ & $-264 \%$ \\
\hline
\end{tabular}


Forecasting future oil production • July 2014

Table 5: Oil import (bbl/day) at a constant consumption of $1.5 \mathrm{M}$ bbl/day for the U.K. and $0.22 \mathrm{M}$ bbl/day for Norway. The import for the E.U. and Norway is a lower bound based on the changes in the U.K and Norway. Negative numbers for Norway represent exports.

\begin{tabular}{lllcc} 
& 2014 & 2020 & 2025 & 2030 \\
\hline Norway & $-1.23 \cdot 10^{6}$ & $-0.88 \cdot 10^{6}$ & $-0.58 \cdot 10^{6}$ & $-0.43 \cdot 10^{6}$ \\
U.K. & $0.7 \cdot 10^{6}$ & $0.9 \cdot 10^{6}$ & $1.0 \cdot 10^{6}$ & $1.1 \cdot 10^{6}$ \\
E.U.+Norway & $9.8 \cdot 10^{6}$ & $10.45 \cdot 10^{6}$ & $10.85 \cdot 10^{6}$ & $11.1 \cdot 10^{6}$ \\
\hline
\end{tabular}

